\title{
Dora Teleky - Ein frühes Mitglied der Deutschen Gesellschaft für Urologie
}

\section{Dora Teleky - an early member of the German Society of Urology}

\author{
Erst seit der Mitte des 19. Jahrhunderts durften Frauen an einigen europäischen Universitäten \\ offiziell ein Studium aufnehmen, davor waren sie fast gänzlich von der wissenschaftlichen Ausbil- \\ dung ausgeschlossen. Dora Teleky (1879/81-1963) war eine der ersten Ärztinnen in Österreich \\ und die erste Frau, die Mitglied der alten Gesellschaft für Urologie (DGfU) war. Ihr Engagement \\ beschränkte sich nicht auf die fachlich-medizinischen Ebene, vielmehr setzte sie sich auch inter- \\ national verstärkt für die Stellung der Ärztinnen in einer von Männern dominierten Medizin ein.
}

\section{Gabriele Possaner von Ehrental: Die erste Ärztin Österreichs als Wegbereiterin \\ $\nabla$}

In der Schweiz waren weibliche Studenten seit 1864, in Frankreich seit 1863 und in Großbritannien seit 1869 zum Studium zugelassen. In Deutschland konnten sich Frauen seit dem WS 1899/1900 an der Freiburger Universität zum Studium einschreiben. Die Vorreiterrolle hatte diesbezüglich Amerika eingenommen, wo die Universitäten bereits seit 1833 für Frauen geöffnet waren [1]. In Österreich durften Frauen an den Philosophischen Fakultäten seit 1897 studieren. Zum Medizinstudium wurden sie aber erst im WS 1900/01 zugelassen. Vor dieser Zeit konnten sie nur im Ausland Medizin studieren [2]. Eine dieser österreichischen Frauen, die im Ausland ihr Studium und ihre Promotion absolvierte, war die Praktische Medizinerin Dr. Gabriele Possanner von Ehrenthal (1860-1940) (• Abb.1). Nach dem erfolgreichen Studium in Genf und Zürich, wo sie auch promovierte, versuchte sie ab 1894 sich in ihrer Heimat niederzulassen. Erst nach etlichen Gesuchen, einer Petition und letztendlich einem stattgegebenen Gnadengesuch an den Kaiser Franz Josef, durfte sie die Rigorosen in Wien ablegen und erlangte am 2. April 1897 als erste Frau in Österreich den medizinischen Doktortitel. Gabriele Possanner war eine streitbare und beharrliche Wegbereiterin für die Zulassung von Frauen an österreichischen Universitäten [3, 4, 5, 6, 7]. Ihr folgten ab 1900 die ersten in Österreich ausgebildeten Ärztinnen. Bereits zehn

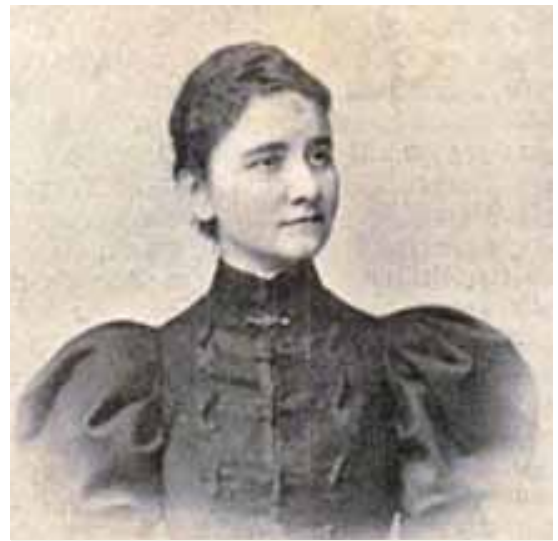

Abb. 1 Dr. Gabriele Possanner von Ehrenthal (1860-1940), Österreichs erste promovierte Ärztin (Bild: Österreichische Nationalbibliothek, NB 515.717, Mit freundlicher Genehmigung).

Jahre später waren in der gesamten k.u.k. Monarchie 80 in Österreich approbierte Ärztinnen tätig. Bedenkt man die Schwierigkeiten, unter denen die Frauen ihr Studium absolvierten und die Skepsis der männlichen Kollegen, so ist es durchaus eine respektable Zahl, die sich im Jahr 1929 auf 477 weibliche Mediziner versechsfachte [5].

\section{Dora Telekys Studien- und Ausbildungsjahre $\nabla$}

Dora Teleky ( $\bullet$ Abb.2) wurde am 5. Juli 1879 oder 1881 in Hinterbrühl/Niederösterreich geboren $[1,8,9]$. Ihr Vater, Hermann Teleky (1837-1921), war ein angesehener Arzt in Wien und auch ihr zehn Jahre älterer Bruder Ludwig (1872-1957)

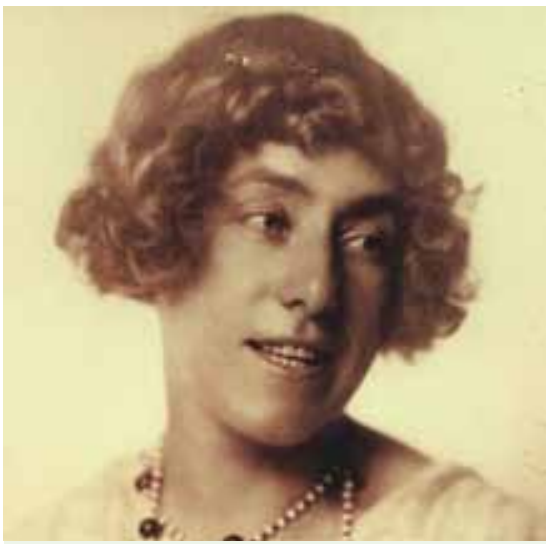

Abb. 2 Dora Teleky (1879/81-1963), erstes weibliches Mitglied der Deutschen Gesellschaft für Urologie. (Museum und Archiv Deutsche Gesellschaft für Urologie, Repro Keyn, Mit freundlicher Genehmigung).

war Mediziner, der sich besonders mit dem Gebiet der Sozialhygiene - mit Schwerpunkt auf den Berufskrankheiten - beschäftigte $[10,11]$.

In ihrem wissenschaftlichen Leben musste Dora Teleky immer wieder Umwege gehen. 1899 legte sie als externer Prüfling ihre Maturaprüfung am Akademischen Gymnasium in Wien ab, nachdem sie zuvor ein privates Mädchen-Gymnasium besucht hatte [12]. Auch ihr Medizinstudium begann Teleky über Umwege, sie wurde als Studentin an der Philosophischen Fakultät an der Wiener Universität immatrikuliert, doch belegte sie Kurse wie Allgemeine Chemie, Anatomie des Menschen und Demonstrationsübungen im Seziersaal. Als Frauen zum Medizin- 
Zusammenfassung

Dora Teleky (1879/81-1963) war eine der ersten Ärztinnen in Österreich und die erste Frau, die Mitglied der alten Gesellschaft für Urologie (DGfU) war. Sie war eine Fachärztin für Gynäkologie, die sich für urologische Themen interessierte, was auch an ihren wissenschaftlichen Arbeiten deutlich wird. Darüber hinaus setzte sie sich auch für berufliche Stellung der Frau in der von Männern dominierten Medizin ein. 1939 emigrierte sie als Reaktion auf den im Jahr zuvor vollzogenen Anschluss Österreichs ans Deutsche Reich in die USA, da sie aus einer jüdischen Familie stammte. Dort war sie weiterhin als Fachärztin für Gynäkologie tätig. Nachdem sie im Ruhestand war, kehrte sie wieder nach Europa zurück und starb 1963 in der Schweiz.

Schlüsselwörter: Geschichte der Urologie -Frauenstudium - jüdische Lebensläufe Urogynäkologie - Wien

\section{Abstract}

Dora Teleky (1879/81-1963) was one of the first female Physicians in Austria and the first woman, who was member of the old Deutsche Gesellschaft für Urologie (German Society of Urology). She was a specialist for gynaecology with great interest in urology, what was reflected in her scientific publications. Furthermore she tried to strengthen the women's position in the male-dominated medicine. In 1939 she emigrated to the USA, because as a Jewish descendant she was pursued after the Austrian "Anschluss" to Germany (Austria's annexation to the German "Third Reich"). There she continued working as specialist for gynaecology. After she had retired, she came back to Europe and died 1963 in Switzerland.

Key Words: history of urology - access for women to universities- Jewish biographies - uro-gynaecology - Vienna

studium zugelassen wurden, wechselte Teleky sofort in dem Semester an die Medizinische Fakultät. Nach dem erfolgreichen Studium in Wien und Straßburg/Elsass wurde sie am 21. Dezember 1904 in Wien promoviert $[2,13]$.

Ihre medizinische Ausbildung setzte Teleky zunächst ein Jahr am Wiener Allgemeinen Krankenhaus im Pathologisch-Anatomischen Institut unter Anton Weichselbaum (1845-1920) als Aspirantin und Sekundärärztin fort. Anschließend war sie bis 1907 als Operationszögling unter Anton von Eiselberg (1860-1939) an der I. Chirurgischen Universitätsklinik tätig. Es folgte ab 1907 bis 1911 die Ausbildung an der II. Frauenklinik unter Rudolf Chrobak (1843-1910), Alfons von Rosthorn (18571909) und Ernst Wertheim (1864-1920). Nach dieser Zeit galt sie als Fachärztin für Gynäkologie und Geburtshilfe. Bis zum Anschluss Österreichs 1938, als die deutsche Reichsärzteverordnung übernommen wurde, gab es keine Verordnung zur Facharztausbildung. Jeder, der sich für fachlich qualifiziert hielt, konnte sich Facharzt nennen. 1920 ließ sie sich mit einer Praxis für Gynäkologie und Geburtshilfe in Wien nieder. Darüber hinaus war sie seit 1919 Leiterin der Schwangeren Fürsorgestelle und seit 1910 bis 1934 die erste Wiener Schulärztin für vier gewerbliche Fortbildungsschulen für Mädchen. $[1,5,6,7,8,9,14]$ Neben ihrer Facharzttätigkeit war sie auch im urologischen Bereich tätig und bildete sich dort weiter.

Die urologische Ausbildung erhielt Dora Teleky von 1911 bis 1914 am Wiener Rothschild-Spital als Volontärin in der Chirurgisch-Urologischen Abteilung unter Otto Zuckerkandel (1861-1921). In dieser Zeit entstanden mehrere Publikationen ( $\bullet$ Tab.1) mit urologischem Bezug und auch ihre Aufnahme in die DGfU fällt in diese Zeit [1]. Als 1914 der Erste Weltkrieg ausbrach, war sie bis 1918 in militärischen Spitälern und Lazaretten tätig und wurde mehrfach für ihre Tätigkeit ausgezeichnet $[1,14]$.

\section{Erste Frau in der DGfU $\nabla$}

Auf dem 3. Kongress der Deutschen Gesellschaft für Urologie in Wien im September 1911 hielt Dora Teleky einen Vortrag mit dem Titel „Teratoider Tumor der weiblichen Harnblase“. Das Thema ihres Vortrags spiegelt ihre urogynäkologische Ausrichtung wider. Sie wurde im gleichen Jahr auch in die Gesellschaft aufgenommen und blieb vermutlich bis 1929 das einzige weibliche Mitglied, die Mitglieds- listen der DGfU verzeichnen außer ihr keine weitere Frau für diese Jahre. Teleky ist auch auf den folgenden Kongressen anwesend. Neun Jahre nach ihrem DGfU-Beitritt, am 20. Januar 1920, trat sie der eben gegründeten Wiener Urologischen Gesellschaft bei.

Doch Dora Teleky war nicht nur im fachlich-medizinischen Bereich engagiert. Sie setzte sich auch verstärkt für die Stellung der Ärztinnen in der männerdominierten Medizin ein. Im Jahr 1919 gründete sie die Organisation der „Ärztinnen Wiens“, deren Vorsitzende sie zehn Jahre lang war. Darüber hinaus war sie korrespondierende Sekretärin des Internationalen Ärztinnenverbandes ( $\bullet$ Abb.3) und nahm am 5. und 6. Kongress der „Medical Women's International Association“ teil. Die Stärkung der Frau sowohl als Patientin als auch als Ärztin war ihr ein wichtiges Anliegen [1, $8,13,14]$.

\section{Schicksalsjahr 1938}

$\nabla$

Dora Teleky stammte aus einer jüdischen Familie und wurde von den antijüdischen Gesetzen nach dem Anschluss Österreichs 1938 aus ihrem Beruf gedrängt, wie auch schon drei ihrer jüdischen Kolleginnen zuvor in Deutschland, die Urologinnen Johanna Hellmann (1890-1981) [15], Helene Fabian-Grumach (1894-1982) [16, 17] und Dora Gerson (1884-1941) [18].

Tab. 1 Urologische Publikationen von Dr. Dora Teleky

1911 „Schädigungen des Harnapparates bei Hebosteotomie und extraperitonealem Kaiserschnitt" (Wiener medizinische Wochenschrift)

1912 „Teratoider Tumor der weiblichen Harnblase“ (Archiv für klinische Chirurgie (Langenbecks Archiv, Berlin)

1912 „Hämaturie bei Frauen“ (Wiener medizinische Wochenschrift)

1914 „Intermittierende Reizblase bei Retroflexio uteri“ (Zeitschrift für Urologie, Berlin)

1922 „Traumatische Striktur der weiblichen Urethra“ (Zeitschrift für urologische Chirurgie, Berlin)

1926 „Vortrag: Ein Fall von Pubertas praecox“ (Wiener medizinische Wochenschrift)

1926 „Über Blasenstörungen bei jungen Mädchen" (Wiener medizinische Wochenschrift)

1930 „Zur Ätiologie und Therapie der weiblichen Reizblase" (Archiv für klinische Chirurgie (Langenbecks Archiv), Berlin) 


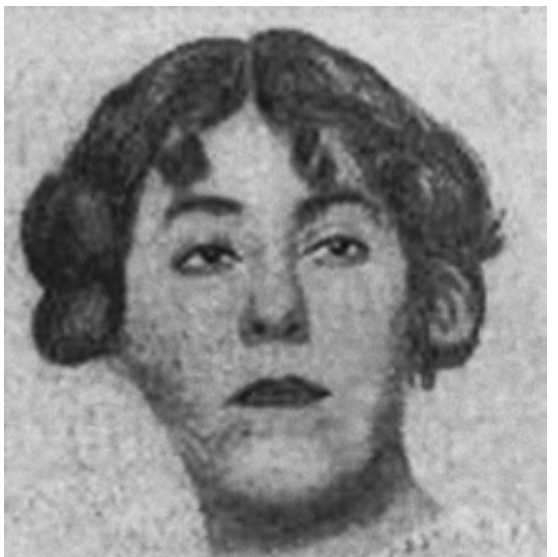

Abb. 3 Dora Teleky in ihrer Zeit als korrespondierende Sekretärin des Internationalen Ärztinnenverbandes (Museum und Archiv Deutsche Gesellschaft für Urologie, Repro Keyn, Mit freundlicher Genehmigung).

Nachdem sich die politische und berufliche Lage durch den Anschluss für Teleky veränderte, entschied sie sich zusammen mit ihrem Mann, Prof. Dr. Ernst von Brücke (1880-1941), in die USA zu emigrieren. Prof. Brücke war seit 1916 Ordinarius für Physiologie in Innsbruck und 1926/27 Rektor. Er war der Enkel des bekannten Wiener Physiologen Ernst Wilhelm von Brücke (1819-1892). Ernst von Brücke galt nach den Nationalsozialistischen $\mathrm{Ge}$ setzen durch seine jüdische Mutter als Halbjude und wurde durch die Eheschließung, vom März 1930, zum Volljuden erklärt und war somit ebenfalls von den antijüdischen Gesetzen betroffen. Nachdem er seine Stellung an der Universität verlor, entschied er sich zu emigrieren und ein Lehrangebot an der Harvard Medical School anzunehmen. Dora folgte ihrem Mann im Herbst 1938 in die USA und reiste über Mailand nach Amerika. Sie erhielt die Zulassung als Gynäkologin in Massachusetts und war bis 1950 noch als Ärztin in Boston tätig. Nachdem sie aus Altersgründen ihren Beruf aufgegeben hatte, kehrte sie nach Europa zurück und starb am 19. April 1963 in Stäfa/Schweiz [1, 5, 13].

\section{Resümee}

$\nabla$

Dora Teleky hatte in mehrfacher Hinsicht eine Vorreiterrolle: Sie war eine der ersten österreichischen Ärztinnen, besuchte regelmäßig nationale und internationale Kongresse, publizierte regelmäßig wissenschaftliche Arbeiten, gilt als erstes weibliches Mitglied der DGfU und der Wiener Urologischen Gesellschaft. Aber auch die Position der Frau als Patientin zu stärken war ihr wichtig. Mit ihren zahlreichen wissenschaftlichen Arbeiten lenkte sie den Blick auf die Behandlung von Frauen in der Urologie. Trotz der einschneidenden Veränderung nach dem Anschluss Österreichs, der erzwungenen Emigration und des plötzlichen Todes ihres Mannes 1941 in Boston, war sie weiterhin als Fachärztin tätig. Dora Telekys Arbeiten im Bereich der Urogynäkologie und ihre Zugehörigkeit zur DGfU als erste Frau sichern ihr bis heute einen festen Platz in der Geschichte der Deutschen Gesellschaft für Urologie und des Faches der Urologie selbst.

\section{Literatur:}

1 Peter JA. Zur Geschichte der ersten Urologinnen in Deutschland [Dissertation]. Hannover. Medizinische Hochschule, 2009

2 Berger E. Das Frauenstudium an der Universität Wien im Zeichen des Liberalismus. www.cajetan.net/forschung_dateien/cfi_Liberalismus_und_Frauenstudium_studie berger.pdf (Recherche 21.11.2011)

3 Gabriele Possanner Institut - Gabriele Possanner Biographie (7.11.2002). www.univie. ac.at/gabriele-possanner-institut/de/gpossanner. (Recherche 3.11.2011)

4 Ariadne - Projekt „Frauen in Bewegung“. Biographie Gabriele Possanner (26.4.2006). www.onb.ac.at/ariadne/vfb/bio_possanner. (Recherche 3.11.2011)

5 Fisch S. Fleiß, Ausdauer, Gewissenhaftigkeit (Teil 1). Ärztewoche 1/2008. www.springermedizin.at/artikel/8485-fleiss-ausdauergewissenhaftigkeit-teil-1 (Recherche 3.11.2011)

6 Arias I. Die ersten Ärztinnen in Wien: Ärztliche Karrieren von Frauen zwischen 1900 und 1938. In: Bolognese-Leuchtenmüller B, Horn S. Hrsg. Töchter des Hippokrates: 100 Jahre akademische Ärztinnen in Österreich. Wien, Verlag der Österreichischen Ärztekammer; 2000: 55-78

7 Horn S, Arias I. Hrsg. Sozialgeschichte der Medizin - Medizinerinnen. Wiener Gespräche. Band 3; Wien, Verlagshaus der Ärzte; 2003

8 Frank M. Doctor for women - Dora Teleky's commitment to gynaeco-urology. J Urol 2010; 183 (Suppl. 4): 434

9 Figdor PP. Ärztinnen in der Urologie Teil 2 Dora Brücke-Teleky (1881-1963). Urologik 2003; 2: 32-33

10 Andreas W. Der Sozialmediziner Ludwig Teleky (1872-1957) und die Entwicklung der Gewerbehygiene zur Arbeitsmedizin. Frankfurt/Main, Mabuse-Verlag 2001

11 Woelk W. Die Westdeutsche Sozialhygienische Akademie in Düsseldorf von 19201933. Aspekte ihrer Geschichte. In: Milles D, Schmacke N, Hrsg. Ludwig Teleky und die Westdeutsche Sozialhygienische Akademie. Schriftenreihe der Akademie für Öffentliches Gesundheitswesen in Düsseldorf. Band 20; Düsseldorf 1999: 55-69
12 Haffner M, Niederkofler H. Etappensiege Frauen in Wissenschaft und Forschung. Sprechende Texte aus der Frauengeschichte. (07.03.2011) www.bmwf.gv.at/fileadmin/ user_upload/gender/Lesetext_100.Frauentag.pdf (Recherche 07.11.2011)

13 Butta-Bieck F. Medizin im Bild / Dora BrückeTeleky. (2011). www.alumni-meduniwien. at/news/medizin+im+bild/dora+bruecketeleky/ (Recherche 07.11.2011)

14 Ariadne - Projekt „Frauen in Bewegung”. Biographie Dora Teleky (21.07.2006). www. onb.ac.at/ariadne/vfb/bio_teleky. (Recherche 02.11.2011)

15 Bellmann J. Lemma Hellmann, Johanna. In: Krischel M et al. Hrsg., Urologen im Nationalsozialismus. Biographien und Materialien. Band 2. Berlin; Hentrich\&Hentrich 2011: $44 \mathrm{f}$.

16 Bellmann J. Lemma Fabian-Grumach, Lene. In: siehe [15]: 35

17 The Central Archives for the History of the Jewish People Jerusalem (CAHJP); Nachlass Ernst Grumach - P 205

18 Bellmann J. Lemma Gerson, Dora. In: siehe [15]: 38f.

\section{Julia Bellmann, Ulm}

\section{Korrespondenz:}

Julia Bellmann

Institut für Geschichte, Theorie und Ethik

der Medizin, Universität Ulm

Frauensteige 6 (Michelsberg)

$89075 \mathrm{Ulm}$

julia.bellmann@uni-ulm.de 